\title{
ERCP using transgastrostomic endoscopy
}

Percutaneous endoscopic gastrostomy (PEG) is now the most common method of enteral nutrition in patients who require long-term tube feeding [1]. Endoscopic retrograde cholangiopancreatography (ERCP) distresses bedridden patients and puts them at risk of aspiration pneumonia [2]. To avoid such risk, we tried transgastrostomic ERCP and related procedures in four cases.

A bedridden woman patient developed choledocholithiasis and cholangitis; she was unable to lie prone because of a fracture of the right upper limb and contracture deformity. First we tried a transgastrostomic examination using a directviewing endoscope (GIF-XQ240; Olympus, Tokyo, Japan). Although we could observe the ampulla of Vater, we could not cannulate into the common bile duct (CBD) ( Fig. 1 a,b). Next we cannulated using an oblique-viewing endoscope (GIF-XK240; Olympus) transgastrostomically; a cholangiogram showed a stone in the distal CBD ( $\mathbf{F i g . 1} \mathbf{c}$, d). However, it was difficult to insert the devices for lithotomy. To observe and cannulate the ampulla, the angle between the duodenal second portion and antrum (between the tip and main shaft of the endoscope) is sharper in a transgastrostomic approach than when using the usual oral route. Thus a side-viewing duodenoscope is needed for lithotomy. To enlarge the PEG stoma (from $8 \mathrm{~mm}$ ) and insert a duodenoscope (JF-260V; diameter 12.6 mm; Olympus), we used a dilator followed by additional gastropexy. Stones were extracted successfully ( Fig. 1 e,f) after an endoscopic sphincterotomy.

We also treated three patients with bile duct cancer with endoscopic biliary stenting via this transgastrostomic approach.

Many patients who have undergone PEG are bedridden and find it difficult to lie prone because of their diseases. Furthermore, ERCP via the oral cavity may distress these patients and may put them at risk of aspiration pneumonia as a complication of the pharyngeal local anesthesia. Thus a transgastrostomic endoscope has several advantages over conventional methods [3,4]. Transgastrostomic ERCP may be recommended in patients having gastrostomy.
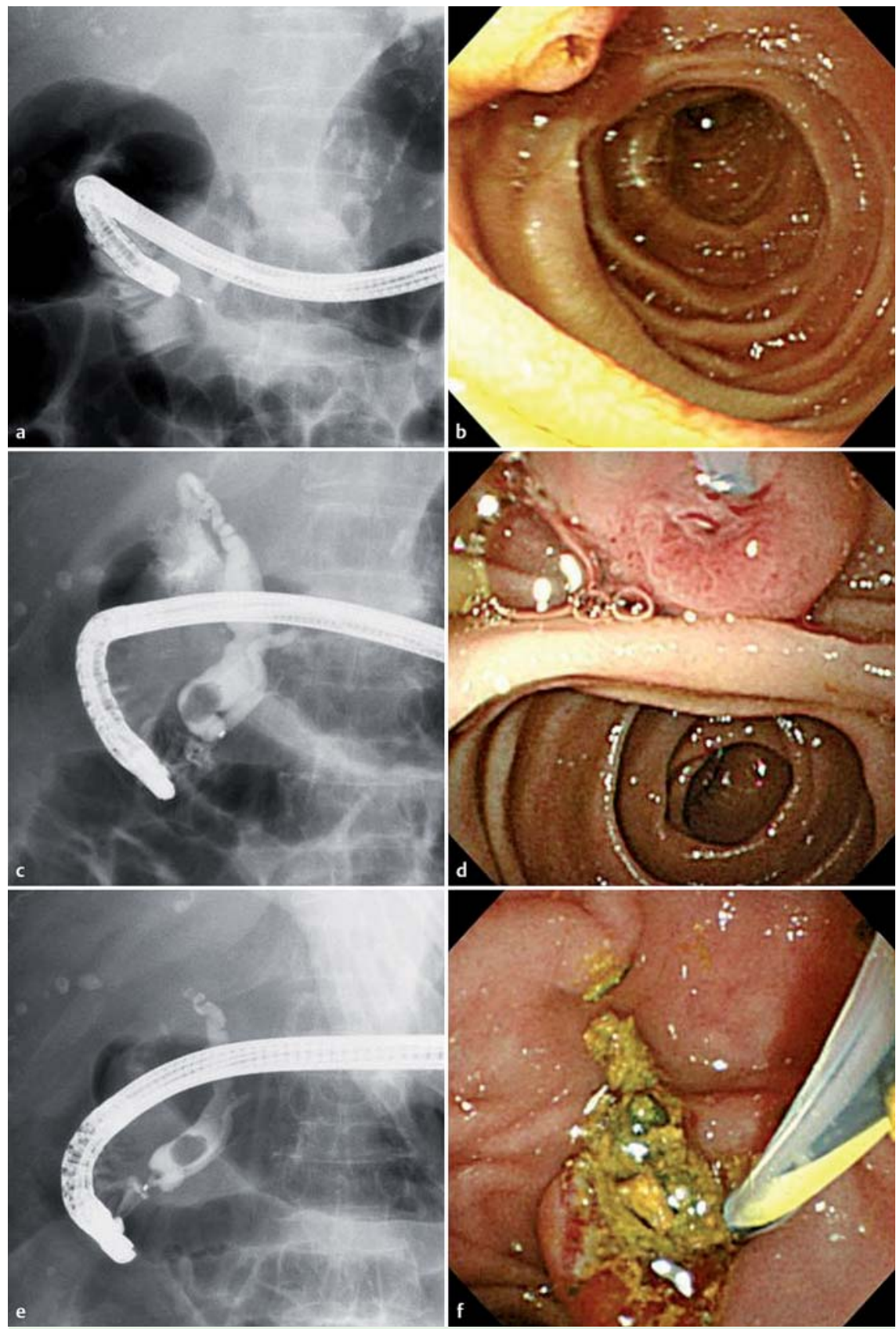

Fig. 1 a In a direct-viewing endoscope, the angle is very acute; $\mathbf{b}$ only observation of the ampulla of Vater is possible. $\mathbf{c}$ Using an oblique-viewing endoscope, a cholangiogram was obtained. $\mathbf{d}$ The degree of bend with an oblique-viewing endoscope, however, is so acute that the devices for lithotomy cannot be inserted into the ampulla. e After dilation of the percutaneous endoscopic gastrostomy (PEG) stoma, a side-viewing duodenoscope was used. $\mathbf{f}$ The bile duct stone was extracted completely using the side-viewing duodenoscope. 
Endoscopy_UCTN_Code_TTT_1AR_2AK

\section{Competing interests: None}

\section{T. Endo ${ }^{1}$, Y. Adachi ${ }^{1}$, H. Mita ${ }^{1}$,}

Y. Arimura ${ }^{2}$

1 Department of Internal Medicine, Sapporo Shirakaba-dai Hospital, Sapporo, Japan

2 Internal Medicine 1, Sapporo Medical University, Sapporo, Japan

\section{References}

1 Suzuki Y, Tamez S, Murakami A et al. Survival of geriatric patients after percutaneous endoscopic gastrostomy in Japan. World J Gastroenterol 2010; 16: 5084-5091

2 Croker JR, Williams SG, Charlton $M$ et al. Endoscopic therapy for bile ducts stones in a geriatric population. Postgrad Med J 1992; 68: $457-460$

3 Imaeda H, Hosoe N, Nakamizo H et al. Clinical results of observation of the upper gastrointestinal tract by transgastrostomic endoscopy using an ultrathin endoscope. J Gastroenterol Hepatol 2010; 25: 1850-1854

4 Nishiwaki S, Araki H, Shirakami Yet al. Transgastrostomic endoscopy-assisted endoscopic submucosal dissection. Endoscopy 2009; 41: E13
Bibliography

DOI $10.1055 / \mathrm{s}-0030-1256932$

Endoscopy 2011; 43: E388 -E389

(c) Georg Thieme Verlag KG Stuttgart · New York . ISSN 0013-726X

\section{Corresponding author}

T. Endo, MD

Department of Internal Medicine Sapporo Shirakaba-dai Hospital

18-2 Tukisamuhigashi Toyohiraku Sapporo 0620052

Japan

Fax: +81-11-8528194

t-endou@kouwakai.or.jp 